\title{
CDK5 suppresses the metastasis of gastric cancer cells by interacting with and regulating $\mathrm{PP2A}$
}

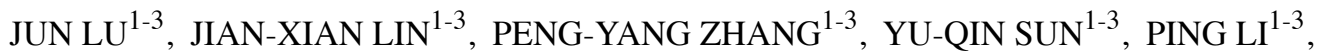 \\ JIAN-WEI XIE ${ }^{1-3}$, JIA-BIN WANG ${ }^{1-3}$, QI-YUE CHEN ${ }^{1,2}$, LONG-LONG CAO $^{1,2}$, \\ YAO LIN ${ }^{4}$, CHANG-MING HUANG ${ }^{1-3}$ and CHAO-HUI ZHENG ${ }^{1-3}$ \\ Departments of ${ }^{1}$ Gastric Surgery and ${ }^{2}$ General Surgery, Fujian Medical University Union Hospital; \\ ${ }^{3}$ Key Laboratory of Ministry of Education of Gastrointestinal Cancer, Fujian Medical University; \\ ${ }^{4}$ College of Life Sciences, Fujian Normal University, Fuzhou, Fujian 350000, P.R. China
}

Received March 16, 2018; Accepted October 16, 2018

DOI: 10.3892/or.2018.6860

\begin{abstract}
Several previous studies have demonstrated that cyclin-dependent kinase (CDK)-5 expression serves an important role in promoting the development of malignant tumours. We have previously reported that CDK5 suppresses gastric tumourigenesis. The aim of the present study was to investigate the mechanistic basis of CDK5. The results of immunoprecipitation and western blot analysis demonstrated that CDK5 could interact with serine/threonine-protein phosphatase 2A (PP2A). The use of an inhibitor of PP2A in CDK5-overexpressing gastric cancer (GC) cell lines antagonized CDK5-mediated suppression in GC cells. Further analysis revealed that PP2A expression was downregulated in GC and patients with low levels of PP2A had worse survival outcomes than those with high levels of PP2A ( $\mathrm{P}=0.035)$. Therefore, the present study provided a novel mechanism for CDK5-mediated tumour suppression, suggesting that CDK5 may be an attractive target for future therapeutic strategies for treating GC. In addition, low levels of PP2A may indicate a tendency for poor prognosis in patients with $\mathrm{GC}$.
\end{abstract}

\section{Introduction}

Gastric cancer (GC) has the fourth highest incidence among all types of cancer and is the third leading cause of cancer-associated mortalities worldwide (1). Despite a steady decline in the incidence and rate of mortality associated with GC due to improved nutrition and the eradication of $H$. pylori in recent years, GC remains a major public health concern (1). Although

Correspondence to: Professor Chao-Hui Zheng or Professor Chang-Ming Huang, Department of Gastric Surgery, Fujian Medical University Union Hospital, 29 Xinquan Road, Fuzhou, Fujian 350000, P.R. China

E-mail: wwkzch@163.com

E-mail: hcmlr2002@163.com

Key words: gastric cancer, cyclin-dependent kinase 5, serine/ threonine-protein phosphatase $2 \mathrm{~A}$, prognosis, therapeutics various treatment options are available, GC has a poor prognosis (2). Human GC tumourigenesis is a multistep and multifactorial process that is associated with several genetic and molecular alterations, including the activation of various oncogenes, inactivation of tumour suppressor genes and abnormal expression of cell cycle-associated proteins (3-6).

The abnormal expression and dysregulation of Cyclin-dependent kinases (CDKs), including CDK1, CDK2, CKD3, CDK4 and CDK6, have recently emerged as important mechanisms underlying the tumourigenesis of certain types of cancer (7-18). However, the role of CDK5 in GC remains relatively unknown. CDK5 is a proline-directed serine/threonine kinase that was first discovered and reported by Hellmich et al in 1992 (19). Unlike the other CDKs, CDK5 has no known cell cycle or mitotic function and is not activated by cyclins (20). Recently, CDK5 activities beyond the nervous system have emerged, and an increasing body of evidence has indicated that CDK 5 may serve a role in cancer tumourigenesis and progression (21-25). Our previous study demonstrated that CDK5 levels decrease in GC and that CDK5 nuclear accumulation suppresses gastric tumourigenesis (26).

Serine/threonine-protein phosphatase 2A (PP2A) is a serine/threonine phosphatase that is comprised of catalytic, scaffold and regulatory subunits. The catalytic and scaffold subunits have 2 isoforms, and the regulatory subunit is derived from 4 different families of isoforms. The regulatory subunit is the most diverse, with temporal and spatial specificity. PP2A dephosphorylates a number of critical cellular molecules, including protein kinase $\mathrm{B}$, mitogen-activated protein kinase kinase (MEK), extracellular signal-regulated kinase (ERK), $\mathrm{p} 53, \mathrm{c}-\mathrm{Myc}$, and $\beta$-catenin; it also regulates a variety of cellular processes, including cell proliferation, signal transduction and apoptosis (27). Aberrant expression, mutations and somatic alterations of PP2A have been associated with the development of human lung (28), breast (29), skin (27) and colon cancers (30). Tsuchiya et al (31) reported that the phosphatidylinositol derivative, 1,2-O-bis-[8-\{2-(2-pentyl-cyclopropylmeth-yl)-cycloprop yl\}-octanoyl]-sn-glycero-3-phosphatidyl-D-1-inositol, serves as an enhancer of PP2A to dephosphorylate and inactivate MEK, thereby inducing the caspase-independent apoptosis of MKN28 human GC cells with high MEK activity. However, the role of 
PP2A in GC metastasis has not been reported. Based on our previous research, it was hypothesized that a functional association between CDK5 and PP2A may affect GC metastasis.

\section{Materials and methods}

Cell culture. The human GC cell line HGC-27 was obtained from the Cell Line Bank, Chinese Academy of Sciences (Shanghai, China). The cell line was verified by polymerase chain reaction (PCR) and cultured without mycoplasma contamination; the species origin was also confirmed by PCR. In addition, the identity of the cell line was authenticated with short tandem repeat profiling. HGC-27 cells were cultured in RPMI-1640 (Gibco; Thermo Fisher Scientific, Inc., Waltham, MA, USA) supplemented with $10 \%$ foetal bovine serum (FBS; Gibco; Thermo Fisher Scientific, Inc.) and incubated at $37^{\circ} \mathrm{C}$ in a humidified atmosphere containing $5 \% \mathrm{CO}_{2}$.

Immunoprecipitation(IP). Cells were washed with ice-cold PBS and lysed in Tris-buffered saline ( $\mathrm{pH}$ 7.4) containing $50 \mathrm{mmol} / 1$ Tris, $150 \mathrm{mmol} / 1 \mathrm{NaCl}, 1 \%$ Nonidet P-40, $1 \mathrm{mmol} / \mathrm{l}$ EDTA, $1 \mathrm{mmol} / 1 \mathrm{Na}_{3} \mathrm{VO}_{4}, 10 \mathrm{mmol} / \mathrm{l} \mathrm{NaF}, 2.5 \mathrm{mg} / \mathrm{ml}$ aprotinin and leupeptin, $1 \mathrm{mmol} / 1$ glycerophosphate plus 4-(2-aminoethyl) benzenesulfonyl fluoride hydrochloride and $10 \mathrm{mmol} / \mathrm{l}$ iodoacetate, on ice for $15 \mathrm{~min}$ prior to removing cellular debris and nuclei by centrifugation at $10,000 \mathrm{x}$ g for $5 \mathrm{~min}$ at $4^{\circ} \mathrm{C}$. Cell lysates were incubated with the corresponding primary antibody CDK5 (cat. no. 2506; Cell Signaling Technology, Inc., Danvers, MA, USA) overnight at $4^{\circ} \mathrm{C}$. Protein A-Sepharose beads (Amersham; GE Healthcare, Chicago, IL, USA) in a

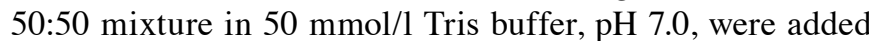
and further incubated for $4 \mathrm{~h}$ at $4^{\circ} \mathrm{C}$. The immunoprecipitates were washed 4 times with Tris-buffered saline and boiled for $5 \mathrm{~min}$ in 401 of Laemmli buffer containing $0.02 \%$ blue bromophenol and $2 \%$ mercaptoethanol.

Western blot analysis. The cells were seeded into $60-\mathrm{mm}$ dishes at a concentration of $5 \times 10^{4}$ cells/well (20-30\% confluence) and cultured to $80 \%$ confluence. The cells were then scraped and lysed in Radioimmunoprecipitation Assay buffer, and the lysates were centrifuged at $10,000 \times \mathrm{g}\left(4^{\circ} \mathrm{C}\right.$ for $10 \mathrm{~min}$ ). Protein concentrations were determined using the BCA Protein Assay kit (Thermo Fisher Scientific, Inc.) according to the manufacturer's instructions. A total of $40 \mu \mathrm{g}$ of protein from each sample was denatured, loaded into each well, separated by $10 \%$ SDS-PAGE, and transferred to a polyvinylidene difluoride membrane (EMD Millipore, Billerica, MA, USA). The membranes were blocked with 5\% non-fat milk at room temperature for $1 \mathrm{~h}$ and then incubated overnight with primary antibodies in TBST (1L TBS buffer with $0.5 \mathrm{ml}$, $0.05 \%$ Tween-20; $1: 1,000)$. Following washing with TBST, the membranes were incubated for $1 \mathrm{~h}$ at room temperature with the corresponding horseradish peroxidase (HRP)-conjugated secondary antibodies at appropriate dilutions and then washed three times with TBST. The proteins on the membranes were visualized using enhanced chemiluminescence (Amersham; GE Healthcare). Primary antibodies against enhanced green fluorescent protein (EGFP; cat. no. 2956; 1:1,000 dilution), CDK5/EGFP-CDK5 (cat. no. 2506; 1:1,000), PP2A/PP2Ac (cat. no. 2038; 1:1,000), Lamin A (cat. no. 86846S; 1:1,000 dilution), a-tubulin (cat. no. 2144; 1:1,000) and GAPDH (cat. no. 5174S; 1:1,000) were purchased from Cell Signaling Technology, Inc. HRP-conjugated goat anti-rabbit immunoglobulin (Ig)-G (cat. no. A4914) and anti-mouse $\operatorname{IgG}$ (cat. no. A0168) were purchased from Sigma-Aldrich; Merck KGaA (Darmstadt, Germany).

Liquid chromatography (LC)-mass spectrometry (MS)/MS and data analysis. A nano-ultra performance (UP)-LC system (Waters Corporation, Milford, MA, USA) was used to separate the peptides. Samples were loaded on a trap column and flushed with mobile phase A $\left(0.1 \%\right.$ formic acid in $\left.\mathrm{H}_{2} \mathrm{O}\right)$ at $5 \mu \mathrm{l} / \mathrm{min}$ for 3 min prior to being delivered onto a nanoUPLC column $(\mathrm{C} 18,150 \times 0.075 \mathrm{~mm}, 1.7 \mu \mathrm{m})$. The peptides were eluted using a $7-45 \%$ B gradient ( $0.1 \%$ formic acid in acetonitrile) over 90 min into a nano-electrospray ionization LTQ Orbitrap mass spectrometer (Thermo Fisher Scientific, Inc.). The mass spectrometer was operated in data-dependent mode in which an initial FT scan recorded the mass range of $350-2,000 \mathrm{~m} / \mathrm{z}$, and the eight most abundant ions were automatically selected for collisional activated dissociation. The spray voltage was set as $2.0 \mathrm{kV}$. The normalized collision energy was set at 35\% for MS/MS. Raw data were compared against the Uniprot human protein database (www.uniprot.org/uniprot/) containing 98,778 sequence entries using the SEQUEST algorithm embedded in the Protein Discoverer 1.3 Software (Thermo Fisher Scientific, Inc.). The following parameters were applied during the database search: $10 \mathrm{ppm}$ precursor mass error tolerance, $1 \mathrm{Da}$ fragment mass error tolerance, static modifications of carbamidomethylation for all cysteine residues, flexible modification of oxidation modifications for methionine residues, and one missed cleavage site of trypsin was allowed. False discovery rate $<0.01$ was used as filtering criteria for all identified peptides. Only proteins identified with $\geq 2$ unique peptides were considered, and proteins identified with the same set of peptides were grouped.

Plasmids and the construction of sTable transfectants for immunofluorescence. EGFP-CDK5-nuclear localisation signal (NLS), EGFP-CDK5-nuclear export signal (NES), EGFP-CDK5-wild-type (WT), pTRE-EGFP, short hairpin (sh)-CDK5 (Lenti-shCKD5) and the corresponding empty vectors Lenti-scramble (Lenti-scr) were constructed, packed and purified by Shanghai GeneChem Co., Ltd. (Shanghai, China) and were transfected into the HGC-27 cell line $\left(5 \times 10^{4}\right.$ cells/well in 6-well plates) with the Xfect Transfection Reagent (cat. nos. 631317 and 631318; Clontech Laboratories, Inc., Mountain View, CA, USA) according to the manufacturer's protocol. EGFP-CDK5-WT plasmids were then introduced using doxycycline (Sigma-Aldrich; Merck KGaA; cat. no. D9891). Then, once selected using puromycin or G418 (Sigma-Aldrich; Merck KGaA), sTable cells were harvested and stored in liquid nitrogen until subsequent use. CKD5 knockdown using Lenti-shCDK5, HGC-27 cells were transfected with shPP2A (lenti-shPP2A) and control plasmid (Shanghai GeneChem Co., Ltd.) according to the manufacturer's protocol using Lipofectamine ${ }^{\mathrm{TM}} 3000$ Transfection Reagent (Thermo Fisher Scientific, Inc.). All of the sTable cells were then stored in liquid nitrogen for subsequent experimentation within 6 months post-transfection. CDK5-overexpressing GC cells 
were treated with Okadaic Acid (Cell Signaling Technology, Inc.; cat. no. 5934) at $100 \mathrm{nM}$ for $30 \mathrm{~min}$ according to the manufacturer's protocol prior to subsequent experiments.

Immunofluorescence. Cells were grown on glass coverslips, washed twice with PBS, fixed with PBS containing $4 \%$ formaldehyde at $4^{\circ} \mathrm{C}$ for $10 \mathrm{~min}$, and permeabilized with $0.2 \%$ Triton X-100 in PBS at $4^{\circ} \mathrm{C}$ for $10 \mathrm{~min}$. Following washing with PBS, cells were blocked with 10\% goat serum (Abcam, Cambridge, MA, USA; cat. no. ab7481) at room temperature for $2 \mathrm{~h}$. Then cells were incubated overnight at $4^{\circ} \mathrm{C}$ with primary antibody against CDK5 (Abcam; cat. no. ab151233; 1:1,000), washed with PBS and incubated with Alexa Fluor $488 \mathrm{IgG}$ (Invitrogen; Thermo Fisher Scientific, Inc; cat. no. A-11008; 1:100; according to the manufacturer's instructions, cells were incubated with $4 \mu \mathrm{g} / \mathrm{ml}$ PBS containing $0.2 \%$ bovine serum albumin for $45 \mathrm{~min}$ at room temperature.). Alexa Fluor $^{\circledR} 568$ phalloidin (Invitrogen; Thermo Fisher Scientific, Inc.) was employed to observe the lamellipodia, and nuclei were stained with DAPI (Sigma-Aldrich; Merck KGaA) at $37^{\circ} \mathrm{C}$ for $5 \mathrm{~min}$. The coverslips were mounted with the SlowFade ${ }^{\circledR}$ Gold reagent (Invitrogen; Thermo Fisher Scientific, Inc.) and observed under a laser confocal scanning microscope.

In vitro cell function experiments. Cell migration (using non-Matrigel coated pore membrane) and invasion assays (using Matrigel coated pore membrane) were performed in Transwell chambers (polycarbonate filters of 8-mm porosity; BD Biosciences, Franklin Lakes, NJ, USA). Cells with serum-free RPMI-1640 were seeded in the upper chamber $\left(5 \times 10^{4}\right.$ cells/well) for $24 \mathrm{~h}$ at $37^{\circ} \mathrm{C}$, then medium in the lower chamber was replaced with complete growth medium containing 5\% FBS (Gibco; Thermo Fisher Scientific, Inc.) and chambers were cultured for $45 \mathrm{~h}$ at $37^{\circ} \mathrm{C}$. Non-invading cells were removed from the upper surfaces of the invasion membranes and the cells on the lower surface were stained with $0.1 \%$ crystal violet for $5 \mathrm{~min}$ at room temperature. Cells at the subcellular level of the microporous membrane were observed and photographed under an optical microscope (magnification, x200).

Colony formation assays were performed to assess the ability of the cells to form colonies. Cell Counting Kit-8 (CCK-8; Dojindo Molecular Technologies, Inc., Kumamoto, Japan) and Sulforhodamine B (SRB; Sigma-Aldrich; Merck KGaA; cat. no. S9012) cell proliferation assays were performed to examine cell viability and cell proliferation. Briefly, cells were seeded into 96-well tissue culture plates at 1,000 cells/well. Plates were incubated for $24,48,72,96$ or $120 \mathrm{~h}$ at $37^{\circ} \mathrm{C}$ with $5 \% \mathrm{CO}_{2}$ in a humidified incubator. Once cells were treated and following incubation, $20 \mu \mathrm{l}$ of CCK-8 was added to each well and cells were further incubated for an additional $3 \mathrm{~h}$. Plates were read on a Biorad ELISA plate reader (Biotek Instruments, Inc., Winooski, VT, USA) using a 450-nm filter. Results of at least three independent experiments were analysed in duplicate. The relative cell proliferation ratios were plotted along with non-treated controls to determine the $100 \%$ level of activity. For the SRB colorimetric assay, cells were seeded into 96-well tissue culture plates at 1,000 cells/well and allowed to adhere for 5 days. Following $24,48,72,96$ or $120 \mathrm{~h}$, cells were harvested and subjected to the SRB protocol, as described previously (32).
Human gastric tumour tissues. GC tissues and the respective adjacent non-tumour tissues were obtained following R0 gastrectomy procedures from 80 patients admitted to Fujian Medical University Union Hospital (Fujian, China) between February 2013 to February 2014 as well as detailed information regarding the clinicopathological parameters. All of the patients underwent R0 gastrectomy. None of the patients underwent preoperative chemotherapy or radiotherapy. Cases with the following characteristics were also excluded: Remnant GC, treated with palliative surgery and concurrent malignancy in other organs. Postoperative adjuvant chemotherapy was performed using 5-fluorouracil-based drugs plus oxaliplatin in advanced cases. The pathological stage of the tumour was reassessed according to the 2010 International Union Against Cancer Tumour-Node-Metastasis (TNM) classification on GC (7th edition) (33). The present study was approved by the Ethics Committee of Fujian Medical University Union Hospital (no. UH-2013009), and written informed consent was obtained from all patients involved.

$R N A$ isolation and reverse transcription-quantitative $P C R$ $(R T-q P C R)$. RNA isolation was performed using TRIzol reagent (Thermo Fisher Scientific, Inc.) according to the manufacturer's manual. RT-PCR was performed with the ReverTra Ace qPCR RT kit (Toyobo Life Science, Osaka, Japan) using oligo-dT-primers and $1 \mu \mathrm{g}$ of mRNA/reaction as a template. The temperature protocol for RT-PCR was as follows: $25^{\circ} \mathrm{C}$ for $5 \mathrm{~min}, 42^{\circ} \mathrm{C}$ for $60 \mathrm{~min}$ and $70^{\circ} \mathrm{C}$ for $5 \mathrm{~min}$. RT-qPCR analysis was performed in triplicate on the ABI PRISM 7500 Sequence Detection System (Thermo Fisher Scientific, Inc.) using the Thunderbird SYBR qPCR Mix (Toyobo Life Science) according to the manufacturer's recommendations. The thermocycling conditions for qPCR were as follows: 40 cycles at $95^{\circ} \mathrm{C}$ for $3 \mathrm{~min}, 95^{\circ} \mathrm{C}$ for $15 \mathrm{sec}$ and $60^{\circ} \mathrm{C}$ for $30 \mathrm{sec}$. The relative expression of PP2A was normalized to GADPH expression levels. The primer sequences were as follows: PP2A, 5'-CGAGAGTCATGACACGGAGG-3' (forward) and 5'-GAGAACGCTTCCATTTGCCC-3' (reverse); and GAPDH: 5'-GGTCGGAGTCAACGGATTTG-3' (forward) and 5'-ATG AGCCCCAGCCTTCTCCAT-3' (reverse). RNA expression levels were obtained using the comparative $2^{-\Delta \Delta C q}$ method (34).

Follow-up. All patients were systematically followed up by trained doctors based on the institutional follow-up protocol using several approaches, including outpatient service, written letters, telephone calls, mail or visitation. Follow-up was conducted every 3 months during the first year and every 6 months from the second year onwards, and all surviving patients were followed for $>3$ years. The survival time was defined as the time from the date of surgery until the date of last contact or mortality. All 80 patients involved in the immunohistochemical analysis were followed up, and none were lost.

Statistical analysis. All statistical analyses were performed using SPSS 18.0 for Windows (SPSS, Inc., Chicago, IL, USA) and GraphPad Prism 5.0 software (GraphPad Software, Inc., La Jolla, CA, USA). Chi-squared tests were used to evaluate differences in proportions. Differences among groups were assessed by one-way analysis of variance 
A

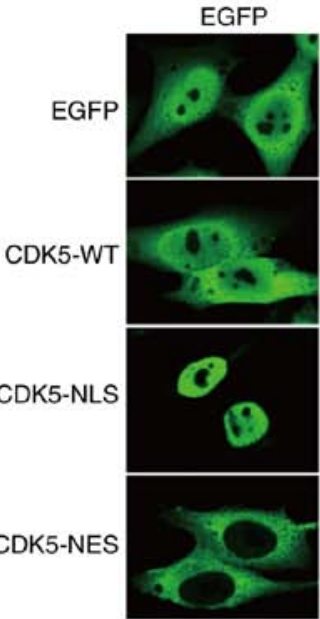

D

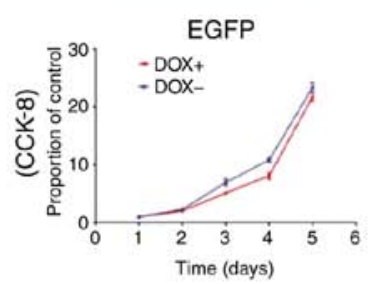

CDK5-NLS

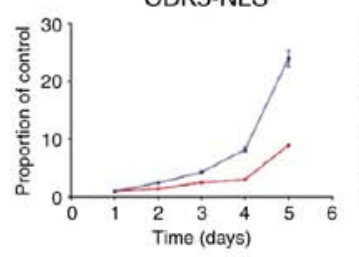

DAPI
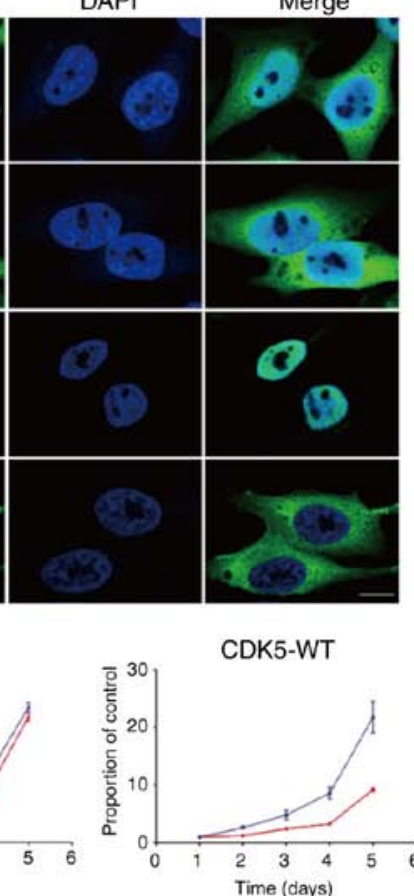

CDK5-NES

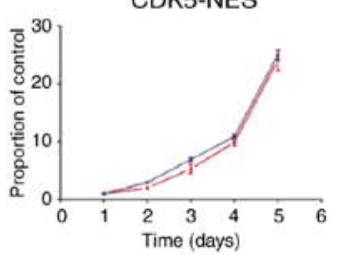

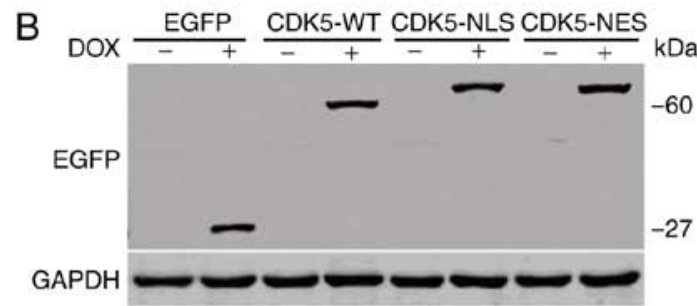

C

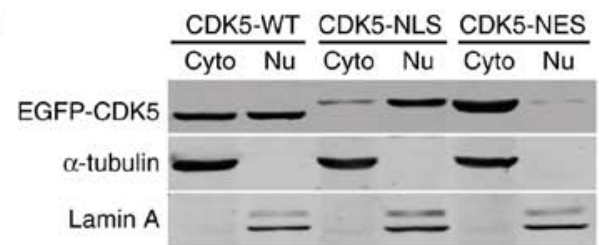

E
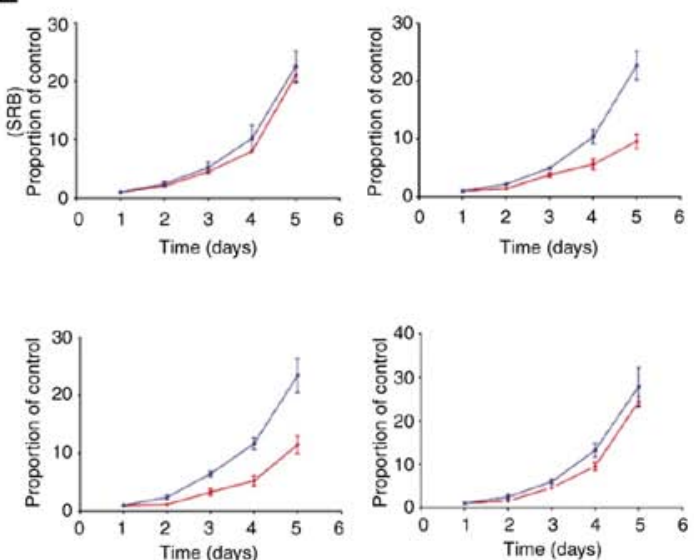

$\mathrm{F}$
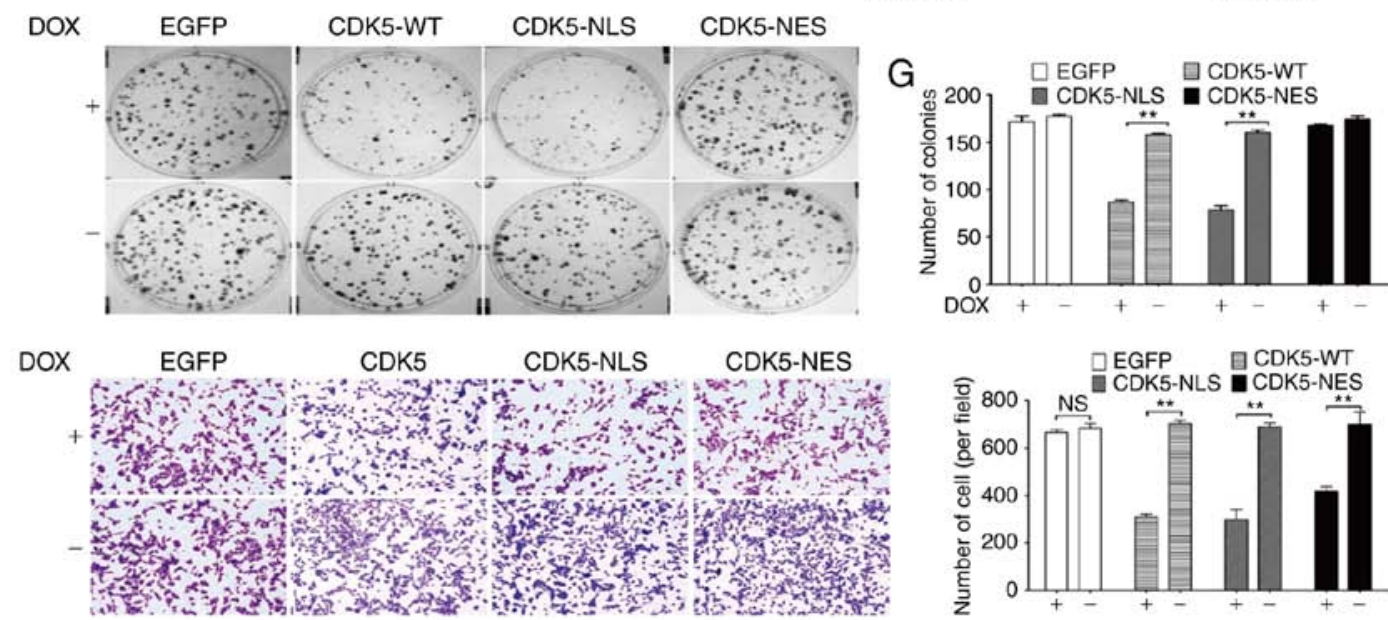

Figure 1. Function of CDK5 in different subcellular locations. (A) HGC-27 cells with stably overexpressed CDK5 in different subcellular locations were generated and examined by immunofluorescence. (B and C) The changes in CDK5 expression (overexpression and knockdown) were confirmed using western blotting. (D) CCK-8 and (E) SRB assays were performed to verify the function of CDK5 in regard to cell proliferation. (F) CDK5 overexpression reduced the colony formation of $\mathrm{HGC}-27 \mathrm{GC}$ cells. (G) CDK5 overexpression reduced the metastasis of $\mathrm{HGC}-27 \mathrm{GC}$ cells. Magnification, $\mathrm{x} 200$. ${ }^{* *} \mathrm{P}<0.01$, as indicated. CDK5, cyclin dependent kinase 5; CCK-8, Cell Counting Kit-8; GC, gastric cancer; EGFP, enhanced green fluorescent protein; WT, wild-type; SRB, Sulforhodamine B; NLS, nuclear localisation signal; NES, nuclear export signal; Nu, nuclear; Cyto, cytoplasm; DOX, doxycycline.

with a Student-Newman-Keuls post hoc test, as previously described (35). Univariate survival analysis was performed using the Kaplan-Meier method, and the curves were compared using the log-rank test. Multivariate analysis was performed using the Cox proportional hazards model to further evaluate all significant prognostic factors that were identified in the univariate analysis. $\mathrm{P}<0.05$ was considered to indicate a statistically significant different; all P-values were two-sided.

\section{Results}

CDK5 overexpression in GC cells inhibits the development of $G C$. To examine the function of CDK5 in different subcellular locations, the present study first overexpressed EGFP-CDK5-WT, EGFP-CDK5-NLS, EGFP-CDK5-NES or p-TRE-EGFP in the HGC-27 GC cell line (Fig. 1A-C). Then, CCK-8 and SRB assays were performed, which 
A
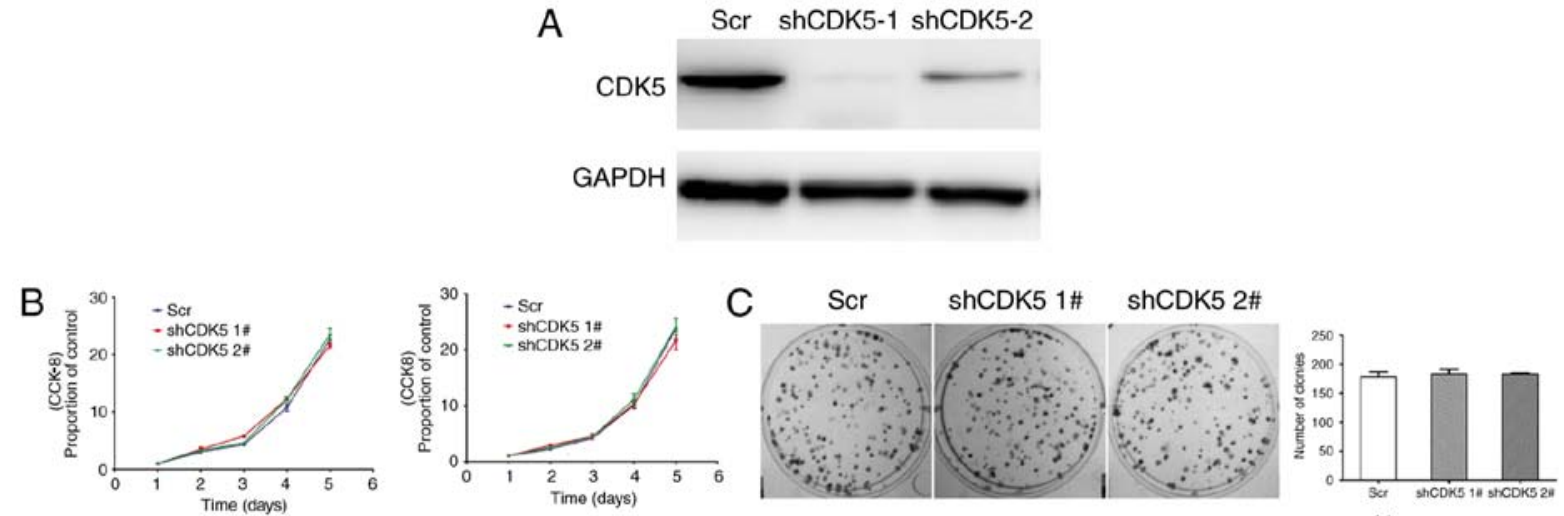

D
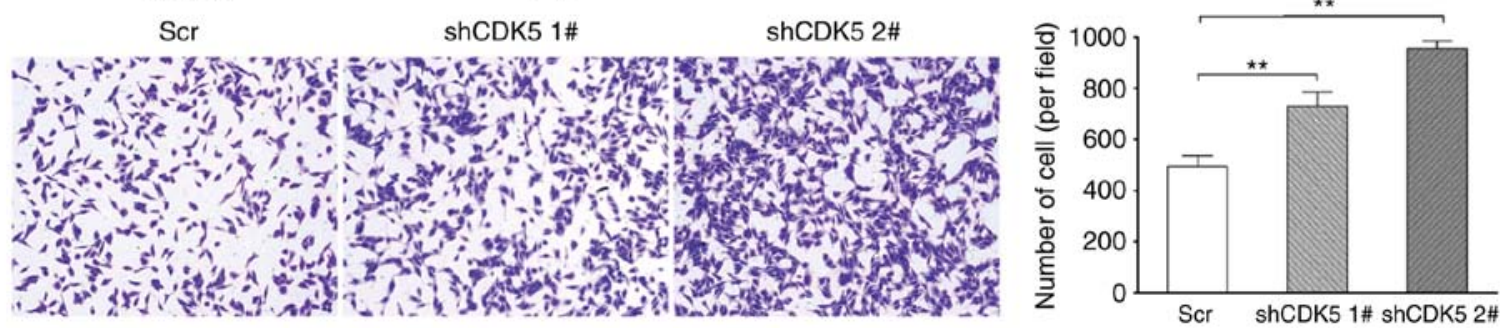

Figure 2. Downregulation of CDK5 in GC cells promotes metastasis. (A) HGC-27 cells with CDK5 stably knocked down and control cells were generated. (B) The proliferation of shCDK5 and control cells was measured by CCK-8 and SRB. (C) Colony formation assays were performed to assess the ability of the shCDK5 and control cells to form colonies. (D) Transwell assays revealed that knockdown of CDK5 in HGC-27 GC cells promoted metastasis. Crystal violet staining. Magnification, x200. ${ }^{* *} \mathrm{P}<0.01$, as indicated. CDK5, cyclin dependent kinase 5; CCK-8, Cell Counting Kit-8; SRB, Sulforhodamine B; GC, gastric cancer; sh-, short hairpin RNA; Scr, scramble.
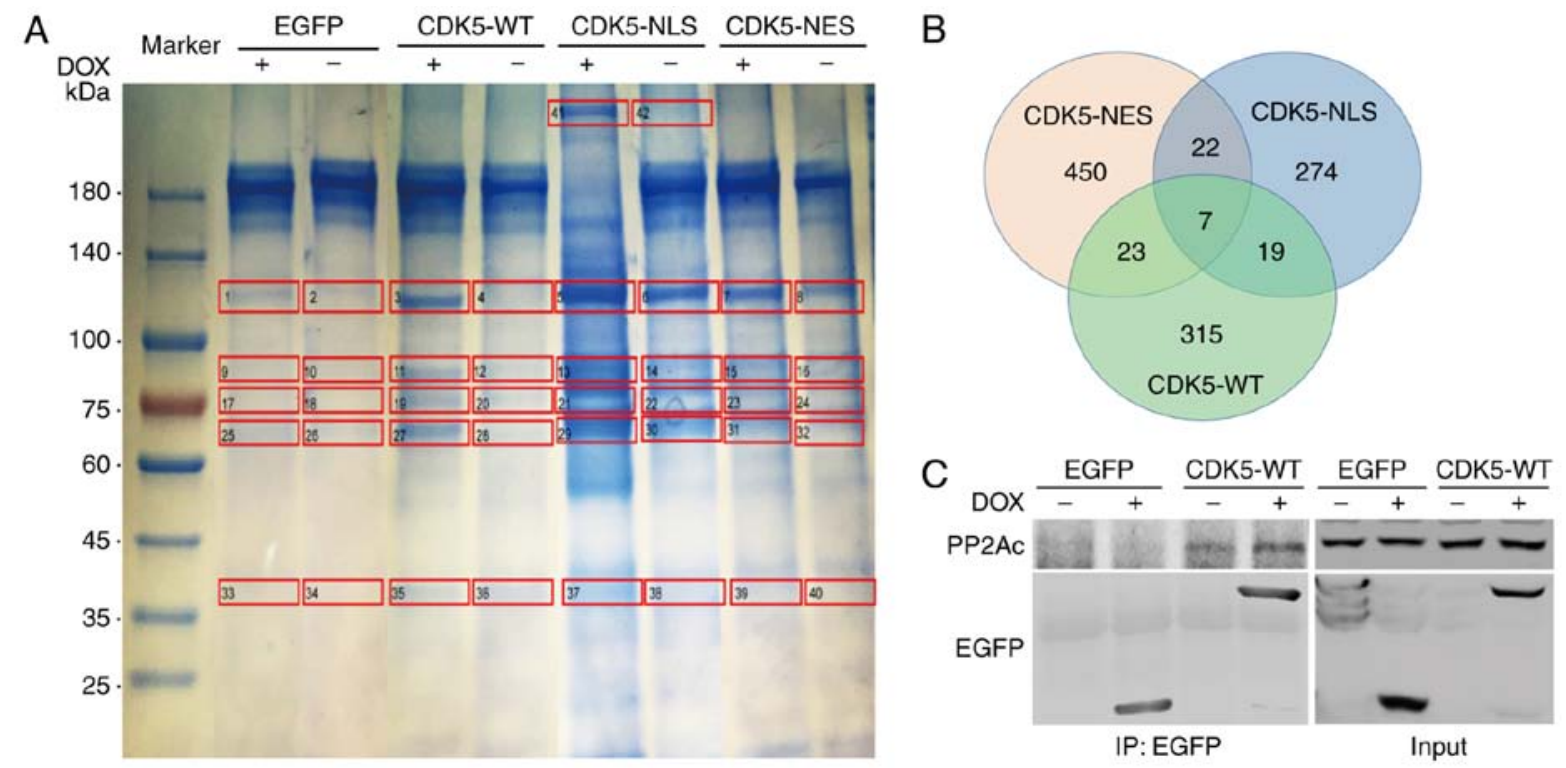

Figure 3. PP2A forms a complex with CDK5. (A and B) IP and Coomassie bright blue staining followed by liquid phase mass spectrometry were performed to identify potential targets of CDK5 in HGC-27 gastric cancer cells. (C) Western blot analysis revealed that CDK5 interacted with PP2A. CDK5, cyclin dependent kinase 5; PP2A, serine/threonine-protein phosphatase 2A; IP, immunoprecipitation; EGFP, enhanced green fluorescent protein; WT, wild-type; NLS, nuclear localisation signal; NES, nuclear export signal; DOX, doxycycline.

revealed that cell proliferation and colony formation were significantly inhibited by nuclear CDK5 expression (Fig. 1D and E). Similarly, nuclear CDK5 expression markedly reduced the colony formation of HGC-27 GC cells and the metastasis of HGC-27 cells in Transwell assays. (Fig. 1F and G). Therefore, in vitro, CDK5 overexpression inhibited HGC-27 cell proliferation, colony formation and metastasis, which was consistent with our previous data obtained with the SGC-7901 and MGC-803 GC cell lines (26).

Downregulation of CDK5 in GC cells promote metastasis. Furthermore, to examine the effect of CDK5 on GC tumourigenicity, HGC-27 cells with CDK5 stably knocked down and control cells were generated (Fig. 2A). In vitro, it was observed that no significant differences were evident 

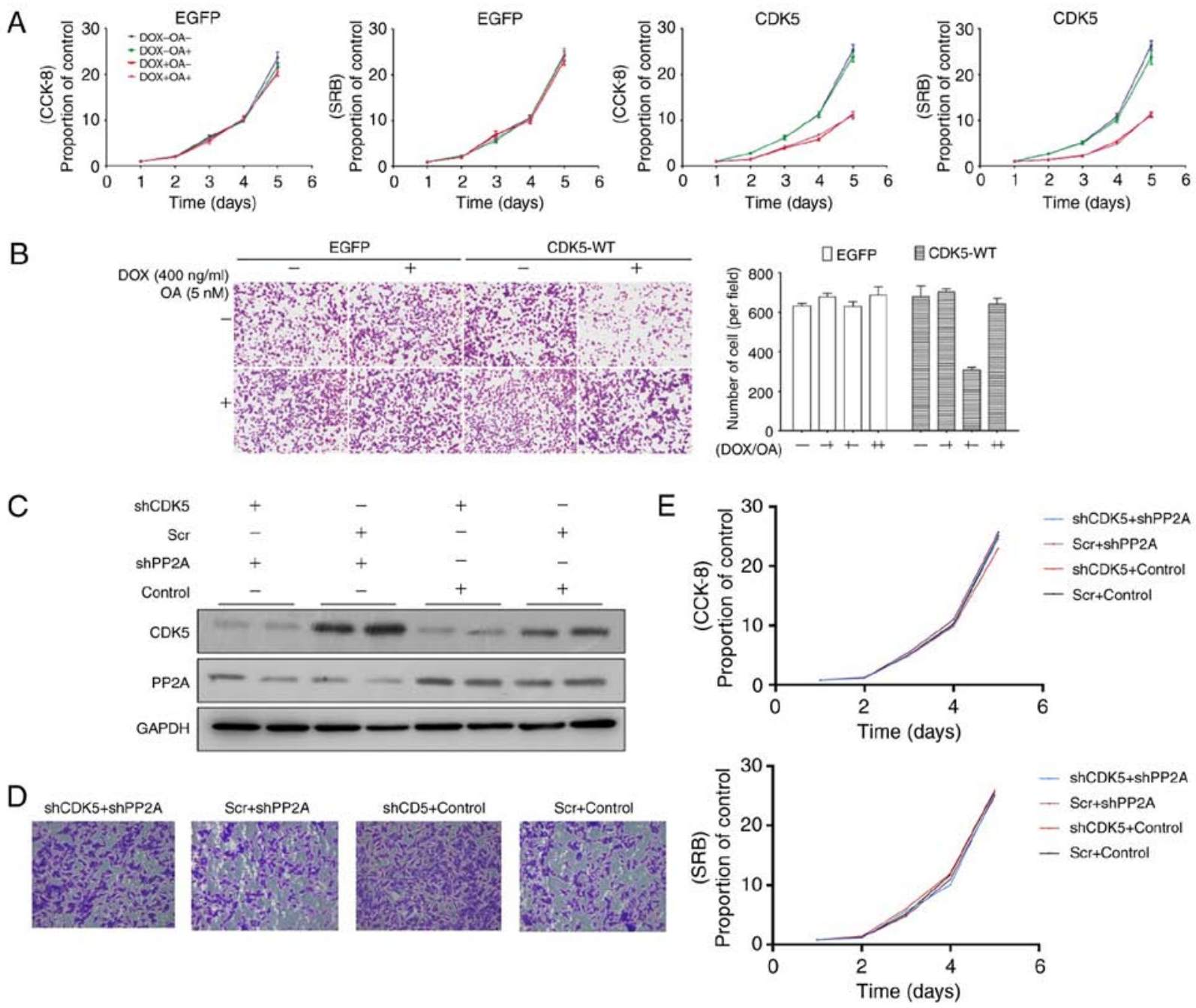

Figure 4. Inhibition of PP2A antagonizes the metastasis of CDK5-overexpressing GC cells. (A) CCK-8 and SRB assays demonstrated that inhibition of PP2A did not significantly affect proliferation. (B) Inhibition of PP2A antagonized the metastasis of CDK5-overexpressing GC cells (magnification, x200), (C) Western blotting of PP2A knockdown in CDK5 knockdown (shCDK5 \#1) HGC-27 cells. (D) Knockdown of PP2A in CDK5 knockdown HGC-27 cells reduced metastasis (magnification, x200). (E) CCK-8 and SRB assays demonstrated that knockdown of PP2A in CDK5 knockdown HGC-27 cells did not significantly affect proliferation. CDK5, cyclin dependent kinase 5; PP2A, serine/threonine-protein phosphatase 2A; CCK-8, Cell Counting Kit-8; GC, gastric cancer; sh-, short hairpin RNA; Scr, scramble; EGFP, enhanced green fluorescent protein; WT, wild-type; SRB, Sulforhodamine B; DOX, doxycycline; OA, Okadaic Acid.

in terms of proliferation and colony formation between the shCDK 5 cells and control cells (Fig. 2B and C). However, the knockdown of CDK5 in HGC-27 GC cells promoted metastasis (Fig. 2D).

Screening and verification of novel protein target of CDK5. To investigate the functional mechanism of CDK5 in GC, the present study performed IP and LC-MS to identify potential targets of CDK5 in HGC-27 GC cells overexpressing EGFP-tagged CDK5-NLS, EGFP-tagged CDK5-NES or p-TRE-EGFP (Fig. 3A). Seven proteins were expressed in all of the groups (Fig. 3B). Further examination by co-IP and western blot analysis revealed that CDK5 interacted with PP2A (Fig. 3C). Therefore, it was hypothesized that CDK5 in combination with PP2A may serve a crucial role in GC.

Inhibition of PP2A antagonizes the metastasis of CDK5-overexpressing $G C$ cells. Next, cells were treated with okadaic acid, an inhibitor of PP2A, and alterations in the proliferation, colony formation and metastasis of HGC-27 GC cells overexpressing CDK5 and in the control group were observed. No significant differences were identified between the two groups in terms of proliferation (Fig. 4A), though a reduction in the metastasis of GC cells overexpressing CDK5 was revealed (Fig. 4B). Furthermore, knockdown of PP2A reduced metastasis (Fig. 4C and D) in CKD5 knockdown (shCDK5 \#1) HGC-27 cells, but it did not affect proliferation (Fig. 4E). Consequently, it was concluded that CDK5 may influence the metastasis of GC by interacting with PP2A.

$P P 2 A$ expression is downregulated in $G C$. In the present study, it was demonstrated that PP2A interacted with CDK5 and subsequently influenced the metastasis of GC. For further analysis, RT-qPCR was performed to examine the relative expression levels of PP2A in 80 pairs of GC tissues matched with normal tissues (Fig. 5A and B). PP2A expression in the $\mathrm{GC}$ tissues was lower than in the corresponding adjacent non-tumour tissues (Fig. 5). 
Table I. Association analyses between expression levels of serine/threonine-protein phosphatase 2A and clinicopathological features in 80 patients with gastric cancer.

\begin{tabular}{|c|c|c|c|c|c|}
\hline \multirow[b]{2}{*}{ Characteristic } & \multicolumn{2}{|c|}{$\begin{array}{c}\text { Low } \\
\text { expression }(n=53)\end{array}$} & \multicolumn{2}{|c|}{$\begin{array}{c}\text { High } \\
\text { expression }(n=27)\end{array}$} & \multirow[b]{2}{*}{ P-value } \\
\hline & $\mathrm{n}$ & $\%$ & $\mathrm{n}$ & $\%$ & \\
\hline Age, years & & & & & 0.055 \\
\hline$<60$ & 27 & 50.9 & 7 & 25.9 & \\
\hline$\geq 60$ & 26 & 49.1 & 20 & 74.1 & \\
\hline Sex & & & & & 0.604 \\
\hline Female & 15 & 28.3 & 6 & 22.2 & \\
\hline Male & 38 & 71.7 & 21 & 77.8 & \\
\hline Tumour location & & & & & 0.339 \\
\hline Upper & 13 & 24.5 & 5 & 18.5 & \\
\hline Middle & 12 & 22.6 & 6 & 22.2 & \\
\hline Lower & 27 & 50.9 & 13 & 48.1 & \\
\hline Mixed & 1 & 1.9 & 3 & 11.1 & \\
\hline Tumour size, mm & & & & & 0.235 \\
\hline$<50$ & 27 & 50.9 & 18 & 66.7 & \\
\hline$\geq 50$ & 26 & 49.1 & 9 & 33.3 & \\
\hline Vascular invasion & & & & & 0.267 \\
\hline Negative & 38 & 71.7 & 23 & 85.2 & \\
\hline Positive & 15 & 28.3 & 4 & 14.8 & \\
\hline Lymphatic invasion & & & & & 0.581 \\
\hline Negative & 39 & 73.6 & 22 & 81.5 & \\
\hline Positive & 14 & 26.4 & 5 & 18.5 & \\
\hline Nervous invasion & & & & & 0.999 \\
\hline Negative & 42 & 79.2 & 22 & 81.5 & \\
\hline Positive & 11 & 20.8 & 5 & 18.5 & \\
\hline Tumour differentiation & & & & & 0.154 \\
\hline Differentiated & 25 & 47.2 & 18 & 66.7 & \\
\hline Undifferentiated & 28 & 52.8 & 9 & 33.3 & \\
\hline $\mathrm{CEA}, \mathrm{ng} / \mathrm{ml}$ & & & & & 0.999 \\
\hline$\leq 5$ & 40 & 75.5 & 20 & 74.1 & \\
\hline$>5$ & 13 & 24.5 & 7 & 25.9 & \\
\hline CA 19-9, IU/ml & & & & & 0.999 \\
\hline$\leq 37$ & 44 & 83.0 & 23 & 85.2 & \\
\hline$>37$ & 9 & 17.0 & 4 & 14.8 & \\
\hline AJCC TNM stage & & & & & 0.429 \\
\hline I & 8 & 15.1 & 2 & 7.4 & \\
\hline II & 19 & 35.8 & 8 & 29.6 & \\
\hline III & 26 & 49.1 & 17 & 63.0 & \\
\hline
\end{tabular}

CEA, carcinoembryonic antigen; CA 19-9, cancer antigen 19-9; AJCC TNM, American Joint Committee on Cancer Tumour-Node-Metastasis.

Associations between PP2A expression and clinicopathological features and prognosis. To analyse the association between PP2A expression and clinicopathological features, specimens were collected from 80 patients with GC. The cohort was divided into groups: Low PP2A expression group and a high PP2A expression group, according to the median PP2A value. No significant differences were observed in terms of the clinical and pathological characteristics between the high- and low-PP2A groups (Table I). To investigate whether PP2A could serve as a prognostic marker, overall survival (OS) curves were plotted using the Kaplan-Meier method according to the PP2A expression level. Patients with low levels of PP2A had a poorer OS rate when compared with those with high levels of expression ( $\mathrm{P}=0.035$; Fig. 6). In addition, to assess 
Table II. Univariate and multivariate Cox proportional analysis for survival.

\begin{tabular}{|c|c|c|c|c|c|}
\hline \multirow[b]{2}{*}{ Parameter } & \multirow[b]{2}{*}{ Categories } & \multicolumn{2}{|c|}{ Univariate analysis } & \multicolumn{2}{|c|}{ Multivariate analysis } \\
\hline & & HR $(95 \% \mathrm{CI})$ & P-value & $\mathrm{HR}(95 \% \mathrm{CI})$ & P-value \\
\hline Age (years) & $(\geq 60$ vs. $<60)$ & $1.616(0.8484-3.081)$ & 0.136 & & \\
\hline Sex & (male vs. female) & $1.165(0.596-2.279)$ & 0.658 & & \\
\hline Tumour location & (upper vs. other) & $1.342(0.545-3.308)$ & 0.522 & & \\
\hline Tumour size & $(\geq 50 \mathrm{~mm}$ vs. $<50 \mathrm{~mm})$ & $1.517(0.826-2.786)$ & 0.179 & & \\
\hline Vascular invasion & (yes vs. no) & $1.998(1.033-3.865)$ & 0.040 & $1.720(0.882-3.352)$ & 0.111 \\
\hline Lymphatic invasion & (yes vs. no) & $1.718(0.876-3.370)$ & 0.115 & & \\
\hline Nervous invasion & (yes vs. no) & $1.585(0.795-3.158)$ & 0.190 & & \\
\hline Tumour differentiation & (undifferentiated vs. differentiated) & $1.447(0.789-2.652)$ & 0.232 & & \\
\hline AJCC TNM stage & (III vs. I/II) & $3.398(1.873-6.163)$ & $<0.001$ & $4.271(2.289-7.969)$ & $<0.001$ \\
\hline CEA level & (above normal vs. normal) & $1.272(0.651-2.485)$ & 0.481 & & \\
\hline CA 19-9 level & (above normal vs. normal) & $1.060(0.470-2.388)$ & 0.889 & & \\
\hline PP2A & (high vs. low) & $0.478(0.234-0.973)$ & 0.042 & $0.317(0.152-0.662)$ & 0.002 \\
\hline
\end{tabular}

CEA, carcinoembryonic antigen; CA 19-9, cancer antigen 19-9; AJCC TNM, American Joint Committee on Cancer Tumour-Node-Metastasis; PP2A, serine/threonine-protein phosphatase 2A; HR, hazard ratio; CI, confidence interval.
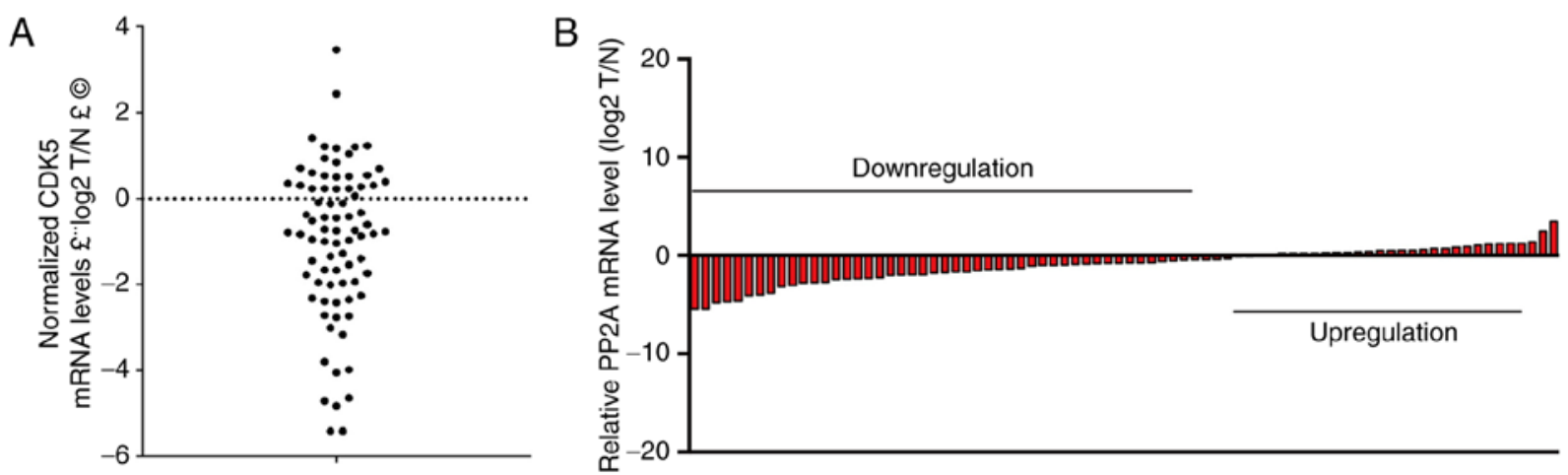

Figure 5. PP2A expression is downregulated in GC. (A and B) Reverse transcription-quantitative polymerase chain reaction was performed to examine the relative expression levels of PP2A in 80 pairs of GC tissues matched with normal tissues. Data are presented as the $\log 2 \mathrm{~T} / \mathrm{N}$. CDK5, cyclin dependent kinase 5; PP2A, serine/threonine-protein phosphatase 2A; GC, gastric cancer; T, gastric cancer tissues; N, normal tissues.

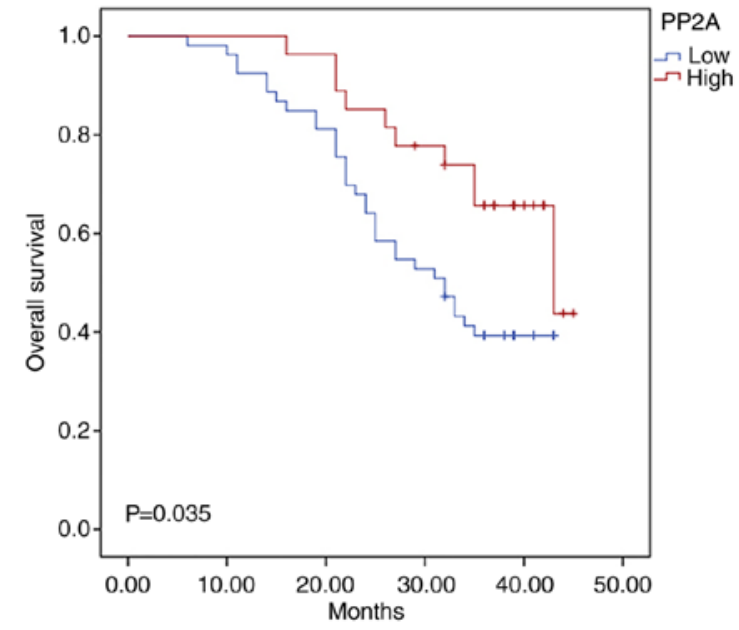

Figure 6. Kaplan-Meier method was used to assess PP2A expression level, which revealed that patients with low levels of PP2A had a poorer overall survival rate than those with high levels of expression $(\mathrm{P}=0.035)$. $\mathrm{PP} 2 \mathrm{~A}$, serine/threonine-protein phosphatase $2 \mathrm{~A}$. whether the use of PP2A to predict OS was independent of the other clinical or pathological factors of the patients with GC, univariate and multivariate Cox proportional hazards analyses were performed. The results revealed that the PP2A levels and TNM stage were independent prognostic factors for OS in patients with GC (Table II). Therefore, low levels of PP2A may be indicative of a poor prognosis in patients with GC.

\section{Discussion}

Recently, many therapeutic strategies have been developed to improve the functional outcomes of GC $(36,37)$. CDK5, a typical CDK that was previously considered to function in processes unrelated to cell cycle regulation, has been shown to serve a fundamental role in several types of carcinomas, including multiple myeloma, and breast, lung, prostate and pancreatic cancers $(22,24,25,38,39)$.

In the present study, the results demonstrated that nuclear CDK5 overexpression markedly reduced the proliferation, 
colony formation and metastasis of HGC-27 GC cells, which was consistent with our previous data obtained with the SGC-7901 and MGC-803 GC cell lines (26). However, CDK5 downregulation was observed to induce metastasis but not proliferation. Zhang et al (40) revealed that CDK5 could function as a cell cycle suppressor when localized to the nucleus of the neuron. To date, the mechanism through which CDK5 regulates the development of $\mathrm{GC}$ is unknown. Based on the present IP and liquid chromatography/MS analysis, it is possible that PP2A may be a potential target of CDK5.

PP2A, a serine/threonine phosphatase, comprises catalytic, scaffold and regulatory subunits and is associated with the development of numerous human cancers (27-30). Therefore, the aim of the present study was to understand whether CDK5 functioned with PP2A during the development of GC. When cells were treated with an inhibitor of PP2A, it was demonstrated that the metastasis of CDK5-overexpressing GC cells was restored without affecting proliferation. When PP2A was knocked down in CKD5 knockdown HGC-27 cells, the results were consistent those aforementioned. Therefore, it was proposed that CDK5 may influence the metastasis of GC by interacting with PP2A. In addition, Tsuchiya et al (31) identified a novel PP2A enhancer that induced the caspase-independent apoptosis of MKN28 GC cells, which is consistent with the present results. Further analysis revealed that relative PP2A expression was downregulated in 80 pairs of matched GC and normal tissues. Based on the median PP2A value, the cohort was divided into groups with low and high PP2A expression, and notably, the patients with high levels of PP2A had better survival outcomes than those with low PP2A expression $(\mathrm{P}=0.035)$. Furthermore, univariate and multivariate $\mathrm{Cox}$ proportional hazards analyses demonstrated that the PP2A level was an independent prognostic factor for OS in patients with GC.

In conclusion, to the best of our knowledge, the present study is the first to report that PP2A may affect metastasis by interacting with CDK5 in GC and that low levels of PP2A may indicate a tendency for poor prognosis in patients with GC.

\section{Acknowledgements}

Not applicable.

\section{Funding}

The present study was supported by the Scientific and Technological Innovation Joint Capital Projects of Fujian Province (grant no. 2016Y9031), Construction Project of Fujian Province Minimally Invasive Medical Center [grant no. (2017)171], The Second Batch of Special Support Funds for Fujian Province Innovation and Entrepreneurship Talents (grant no. 2016B013), QIHANG funds of Fujian Medical University (grant no. 2016QH025) and the Science Foundation of the Fujian Province, China (grant no. 2018J01307).

\section{Availability of data and materials}

The datasets analysed during the present study are available from the corresponding author upon reasonable request.

\section{Authors' contributions}

JL conceived and designed the present study. YL and CMH contributed to the design of the present study. JL, YQS and PYZ performed the in vitro experiments. PL, JWX, JBW, and QYC obtained the tumor samples and the clinical data. JXL and LLC analysed the data. JL, PYZ and CHZ wrote and revised the manuscript. $\mathrm{CHZ}$ and $\mathrm{CMH}$ were also involved in the conception of the study. All authors read and approved the manuscript and agree to be accountable for all aspects of the research in ensuring that the accuracy or integrity of any part of the work are appropriately investigated and resolved.

\section{Ethics approval and consent to participate}

The present study was approved by the Ethics Committee of Fujian Medical University Union Hospital (Fujian, China; no. UH-2013009), and written informed consent was obtained from all patients involved.

\section{Patient consent for publication}

Not applicable.

\section{Competing interests}

The authors declare that they have no competing interests.

\section{References}

1. Torre LA, Bray F, Siegel RL, Ferlay J, Lortet-Tieulent J and Jemal A: Global cancer statistics, 2012. CA Cancer J Clin 65: 87-108, 2015.

2. Chen H, Guan R, Lei Y, Chen J, Ge Q, Zhang X, Dou R, Chen H, Liu H, Qi X, et al: Lymphangiogenesis in gastric cancer regulated through Akt/mTOR-VEGF-C/VEGF-D axis. BMC Cancer 15: $103,2015$.

3. Johnson SM and Evers BM: Translational research in gastric malignancy. Surg Oncol Clin N Am 17: 323-340, 2008.

4. Kim K, Chun KH, Suh PG and Kim IH: Alterations in cell proliferation related gene expressions in gastric cancer. Crit Rev Eukaryot Gene Expr 21: 237-254, 2011.

5. Kim DH: Prognostic implications of cyclin B1, p34cdc2, p2 $27^{\mathrm{Kipl}}$ and p53 expression in gastric cancer. Yonsei Med J 48: 694-700, 2007.

6. Liang B, Wang S, Yang X, Ye Y, Yu Y and Cui Z: Expressions of cyclin $\mathrm{E}$, cyclin dependent kinase 2 and $\mathrm{p} 57^{\mathrm{KIP} 2}$ in human gastric cancer. Chin Med J 116: 20-23, 2003.

7. Malumbres M and Barbacid M: Cell cycle, CDKs and cancer: A changing paradigm. Nat Rev Cancer 9: 153-166, 2009.

8. Lapenna S and Giordano A: Cell cycle kinases as therapeutic targets for cancer. Nat Rev Drug Discov 8: 547-566, 2009.

9. Wright RH, Castellano G, Bonet J, Le Dily F, Font-Mateu J, Ballaré C, Nacht AS, Soronellas D, Oliva B and Beato M: CDK2-dependent activation of PARP-1 is required for hormonal gene regulation in breast cancer cells. Genes Dev 26: 1972-1983, 2012 .

10. Yang S, Zhang L, Liu M, Chong R, Ding SJ, Chen Y and Dong J: CDK1 phosphorylation of YAP promotes mitotic defects and cell motility and is essential for neoplastic transformation. Cancer Res 73: 6722-6733, 2013.

11. Cepeda D, Ng HF, Sharifi HR, Mahmoudi S, Cerrato VS Fredlund E, Magnusson K, Nilsson H, Malyukova A, Rantala J, et al: CDK-mediated activation of the $\operatorname{SCF}^{\mathrm{FBXO}}$ (28) ubiquitin ligase promotes MYC-driven transcription and tumourigenesis and predicts poor survival in breast cancer. EMBO Mol Med 5: 1067-1086, 2013.

12. Lu M, Breyssens H, Salter V, Zhong S, Hu Y, Baer C, Ratnayaka I, Sullivan A, Brown NR, Endicott J, et al: Restoring p53 function in human melanoma cells by inhibiting MDM2 and cyclin B1/CDK1-phosphorylated nuclear iASPP. Cancer Cell 30: 822-823, 2016. 
13. Radomska HS, Alberich-Jordà M, Will B, Gonzalez D, Delwel R and Tenen DG: Targeting CDK1 promotes FLT3-activated acute myeloid leukemia differentiation through $\mathrm{C} / \mathrm{EBP} \alpha$. J Clin Invest 122: 2955-2966, 2012

14. Sheppard KE and McArthur GA: The cell-cycle regulator CDK4: An emerging therapeutic target in melanoma. Clin Cancer Res 19: 5320-5328, 2013.

15. Rader J, Russell MR, Hart LS, Nakazawa MS, Belcastro LT, Martinez D, Li Y, Carpenter EL, Attiyeh EF, Diskin SJ, et al: Dual CDK4/CDK6 inhibition induces cell-cycle arrest and senescence in neuroblastoma. Clin Cancer Res 19: 6173-6182, 2013.

16. Sakaue-Sawano A, Kurokawa H, Morimura T, Hanyu A, Hama H, Osawa H, Kashiwagi S, Fukami K, Miyata T, Miyoshi H, et al: Visualizing spatiotemporal dynamics of multicellular cell-cycle progression. Cell 132: 487-498, 2008.

17. Tetsu $\mathrm{O}$ and McCormick F: Proliferation of cancer cells despite CDK2 inhibition. Cancer Cell 3: 233-245, 2003.

18. van den Heuvel S and Harlow E: Distinct roles for cyclindependent kinases in cell cycle control. Science 262: 2050-2054, 1993.

19. Hellmich MR, Pant HC, Wada E and Battey JF: Neuronal cdc2-like kinase: A cdc2-related protein kinase with predominantly neuronal expression. Proc Natl Acad Sci USA 89: 10867-10871, 1992.

20. Dhavan R and Tsai LH: A decade of CDK5. Nat Rev Mol Cell Biol 2: 749-759, 2001

21. Strock CJ, Park JI, Nakakura EK, Bova GS, Isaacs JT, Ball DW and Nelkin BD: Cyclin-dependent kinase 5 activity controls cell motility and metastatic potential of prostate cancer cells. Cancer Res 66: 7509-7515, 2006.

22. Pozo K, Castro-Rivera E, Tan C, Plattner F, Schwach G, Siegl V, Meyer D, Guo A, Gundara J, Mettlach G, et al: The role of Cdk5 in neuroendocrine thyroid cancer. Cancer Cell 24: 499-511, 2013.

23. Ehrlich SM, Liebl J, Ardelt MA, Lehr T, De Toni EN, Mayr D, Brandl L, Kirchner T, Zahler S, Gerbes AL and Vollmar AM: Targeting cyclin dependent kinase 5 in hepatocellular carcinoma-A novel therapeutic approach. J Hepatol 63: 102-113, 2015

24. Feldmann G, Mishra A, Hong SM, Bisht S, Strock CJ, Ball DW, Goggins M, Maitra A and Nelkin BD: Inhibiting the cyclindependent kinase CDK5 blocks pancreatic cancer formation and progression through the suppression of Ras-Ral signaling. Cancer Res 70: 4460-4469, 2010.

25. Demelash A, Rudrabhatla P, Pant HC, Wang X, Amin ND, McWhite CD, Naizhen X and Linnoila RI: Achaete-scute homologue-1 (ASH1) stimulates migration of lung cancer cells through Cdk5/p35 pathway. Mol Biol Cell 23: 2856-2866, 2012.

26. Cao L, Zhou J, Zhang J, Wu S, Yang X, Zhao X, Li H, Luo M, Yu Q, Lin G, et al: Cyclin-dependent kinase 5 decreases in gastric cancer and its nuclear accumulation suppresses gastric tumorigenesis. Clinical Cancer Res 21: 1419-1428, 2015.

27. Seshacharyulu P, Pandey P, Datta K and Batra SK: Phosphatase: PP2A structural importance, regulation and its aberrant expression in cancer. Cancer Lett 335: 9-18, 2013.

28. Zhou X, Updegraff BL, Guo Y, Peyton M, Girard L, Larsen JE, Xie XJ, Zhou Y, Hwang TH, Xie Y, et al: PROTOCADHERIN 7 acts through SET and PP2A to potentiate MAPK signaling by EGFR and KRAS during lung tumorigenesis. Cancer Res 77: 187-197, 2017.
29. Baldacchino S, Saliba C, Petroni V, Fenech AG, Borg N and Grech G: Deregulation of the phosphatase, PP2A is a common event in breast cancer, predicting sensitivity to FTY720. EPMA J 5: 3, 2014.

30. Cristóbal I, Manso R, Rincón R, Caramés C, Senin C, Borrero A, Martínez-Useros J, Rodriguez M, Zazo S, Aguilera O, et al: PP2A inhibition is a common event in colorectal cancer and its restoration using FTY720 shows promising therapeutic potential. Mol Cancer Ther 13: 938-947, 2014.

31. Tsuchiya A, Kanno T, Shimizu T, Nakao S, Tanaka A, Tabata C, Nakano T and Nishizaki T: A novel PP2A enhancer induces caspase-independent apoptosis of MKN28 gastric cancer cells with high MEK activity. Cancer Lett 347: 123-128, 2014.

32. Lombardo Y, Filipović A, Molyneux G, Periyasamy M, Giamas G, Hu Y, Trivedi PS, Wang J, Yagüe E, Michel L and Coombes RC: Nicastrin regulates breast cancer stem cell properties and tumor growth in vitro and in vivo. Proc Natl Acad Sci USA 109: 16558-16563, 2012.

33. Edge S, Byrd DR, Compton CC, Fritz AG, Greene F and Trotti A (eds): AJCC Cancer Staging Handbook: From the AJCC Cancer Staging Manual. 7th edition. Springer-Verlag, New York, 2010.

34. Livak KJ and Schmittgen TD: Analysis of relative gene expression data using real-time quantitative PCR and the $2^{-\Delta \Delta C \mathrm{~T}}$ method. Methods 25: 402-408, 2001.

35. Lei W, Wang ZL, Feng HJ, Lin XD, Li CZ and Fan D: Long non-coding RNA SNHG12 promotes the proliferation and migration of glioma cells by binding to HuR. Int J Oncol 53: 1374-1384, 2018.

36. Li Y, Gong J, Zhang Q, Lu Z, Gao J, Li Y, Cao Y and Shen L: Dynamic monitoring of circulating tumour cells to evaluate therapeutic efficacy in advanced gastric cancer. Br J Cancer 114: 138-145, 2016.

37. Fujiwara Y, Okada K, Omori T, Sugimura K, Miyata H, Ohue M, Kobayashi S, Takahashi H, Nakano H, Mochizuki C, et al: Multiple therapeutic peptide vaccines for patients with advanced gastric cancer. Int J Oncol 50: 1655-1662, 2017.

38. Liang Q, Li L, Zhang J, Lei Y, Wang L, Liu DX, Feng J, Hou P, Yao R, Zhang Y, et al: CDK5 is essential for TGF- $\beta 1$-induced epithelial-mesenchymal transition and breast cancer progression. Sci Rep 3: 2932, 2013.

39. Hsu FN, Chen MC, Lin KC, Peng YT, Li PC, Lin E, Chiang MC, Hsieh JT and Lin H: Cyclin-dependent kinase 5 modulates STAT3 and androgen receptor activation through phosphorylation of $\mathrm{Ser}^{727}$ on STAT3 in prostate cancer cells. Am J Physiol Endocrinol Metab 305: E975-E986, 2013.

40. Zhang J, Li H, Yabut O, Fitzpatrick H, D'Arcangelo G and Herrup K: Cdk5 suppresses the neuronal cell cycle by disrupting the E2F1-DP1 complex. J Neurosci 30: 5219-5228, 2010.

This work is licensed under a Creative Commons Attribution-NonCommercial-NoDerivatives 4.0 International (CC BY-NC-ND 4.0) License. 